\title{
Free-Volume Studies in \\ Polycaprolactone/Poly(propylene oxide) Urethane/Urea Membranes by Positron Lifetime Spectroscopy
}

\author{
M.F. Ferreira Marques ${ }^{a, b, *}$, P.M. Gordo ${ }^{b}$, A.P. De Lima $^{b}$, \\ D.P. Queiroz ${ }^{c}$, M. Norberta De Pinho ${ }^{c}$, P. Major ${ }^{d}$ \\ AND Zs. KAJCSOS ${ }^{d}$ \\ ${ }^{a}$ Department of Chemical Engineering, Instituto Superior de Engenharia \\ 3031-199 Coimbra, Portugal \\ ${ }^{b}$ ICEMS, Department of Physics, University of Coimbra \\ 3004-516 Coimbra, Portugal \\ ${ }^{c}$ Department of Chemical Engineering, Instituto Superior Técnico \\ 1049-001 Lisboa, Portugal \\ ${ }^{d}$ KFKI Research Institute for Particle and Nuclear Physics \\ P.O.B. 49, 1525 Budapest, Hungary
}

\begin{abstract}
Positron annihilation lifetime spectroscopy was used to study the free volume parameters in polypropylene oxide-based tri-isocyanate terminated prepolymer/polycaprolactone diol bi-soft urethane/urea membranes (PU/PCL) with PCL content from 5 up to $25 \mathrm{wt} \%$. Positron annihilation lifetime spectroscopy measurements carried out in the temperature range 298-324 K mirrored the phase separation of the various soft and hard segments present in the membranes. The size and amount of free volume holes determined by positron annihilation lifetime spectroscopy appear to be correlated with the $\mathrm{CO}_{2}$ gas permeability through the membranes.
\end{abstract}

PACS numbers: 61.41.+e, 78.70.Bj, 82.30.Gg

\section{Introduction}

Segmented polyurethanes are a class of synthetic polymers widely used in the medical field, due to their high biocompatibility especially as biomaterials in contact with blood [1]. They usually exhibit a two-phase microstructure, which is caused by the physical differences between the soft and the hard segments.

${ }^{*}$ corresponding author; e-mail: fatima@lipc.fis.uc.pt 
In recent years, positron annihilation lifetime spectroscopy (PALS) has gained growing interest as a useful tool to probe the microscopic properties of polymeric materials, predominantly to investigate free-volume distributions in various crystalline and amorphous solids [2-7]. In polymers, the positron may form and annihilate from a bound state called positronium (Ps) that will be localised in the free-volume holes. The primary annihilation mechanism of the long-lived triplet state of the positronium $(o-\mathrm{Ps})$ is a pick-off annihilation with electrons of the host medium. The $o$-Ps lifetime is sensitive to both the electron density in the neighbourhood of the Ps and to the free-volume hole size [2-7]. For an $o$-Ps confined in an infinitely deep potential well the semiempirical Tao-Eldrup model and its extensions [8-12] establish a correlation between the free-volume hole radius, $R$, and the $o$-Ps pick-off lifetime, $\tau_{\mathrm{o}-\mathrm{Ps}}$, giving $\tau_{\mathrm{o}-\mathrm{Ps}}=0.5\left[1-R / R_{0}+(1 / 2 \pi) \sin \left(2 \pi R / R_{0}\right)\right]^{-1}$, where $R_{0}=R+\Delta R$ and $\Delta R=1.656 \AA$. The average radius $R$ of the free-volume holes in polymers and the average volume of the holes, $V_{\mathrm{f}}=(4 / 3) \pi R^{3}$, can be calculated from the measured $o$-Ps lifetime. The fractional free volume, FFV, is a product of the free volume $V_{\mathrm{f}}$ and positronium intensity $I$, as $\mathrm{FFV}=C V_{\mathrm{f}} I$, where $\mathrm{C}$ is a constant which is empirically estimated from conventional free-volume theory. It must be underlined here that this model has principal limitations (e.g. [13]) as this apparent free volume integrates only the sites seen by the $o$-Ps. However, this model has been successfully applied to many systems, including membranes [14, 15].

The present work is an extension of studies of polyurethanes/urea membranes with two soft segments $[14,16,17]$. Our aim was to study how the freevolume properties are influenced by phase separation and how the free volume correlates with gas permeability.

\section{Experimental}

Cross-linked polyurethane membranes with two soft segments were prepared by extending a polypropylene oxide-based tri-isocyanate terminated prepolymer (PU), supplied by Hoechst Portugal, with polycaprolactone diol (PCL) from Aldrich. Dibutyltin dilauryate (DBTDL), supplied by Aldrich, was used as a catalyst with toluene solvent (Merck).

Our fast-fast coincidence PALS setup with Pilot-U scintillators and XP 2020Q photomultipliers [17] had a time resolution of 270 ps. Sample-source sandwiches with ${ }^{22} \mathrm{Na}$ of $c a$. $0.5 \mathrm{MBq}$ activity closed between $\mathrm{Ni}$ foils were employed. All measurements were carried out in the temperature range of 298-324 K, well above the $T_{\mathrm{g}}$ of the present membranes $\left(223<T_{\mathrm{g}}<233 \mathrm{~K}\right)$. The lifetime spectra (LS) had an integral number of $c a .3 .0 \times 10^{6}$ counts and were evaluated applying the LT (version 9) program [18].

\section{Results and discussion}

The LS data were deconvoluted into three and four lifetime components with lifetimes $\tau_{i}$ and intensities $I_{i}$. The four component analysis proved to be more 
consistent along the concentration range. Subscripts $i=1$ to 4 are ascribed to singlet states of positronium ( $p$-Ps), free positrons, and two long-lived triplet states (o-Ps) associated with pick-off annihilation in free-volume holes of two different sizes, respectively. The latter components could indicate the coexistence of two phases, corresponding to separated domains of the two soft segments (polypropylene oxide and polycaprolactone) present in the membranes. Such results were also observed in other two polyurethanes/urea membranes obtained through the introduction of two soft segments - polypropylene oxide/polybutadiene and polypropylene oxide/polydimetylsiloxane [16, 17].

In Fig. 1a, lifetime $\tau_{4}$ decreases slightly with increasing PCL content and increases with temperature, resulting in a similar dependence for the $R_{4}$ radius. Increasing the temperature in the samples brings about a systematic increase in the average volume of the holes, because, as expected, the chain movements above $T_{\mathrm{g}}$ are no longer frozen. By increasing the temperature, the thermal movements also increase. The lifetime $\tau_{3}$ also decreases below $15 \mathrm{wt} \%$ PCL, remaining almost constant for higher values. However, the temperature affects differently the intensities $I_{3}$ and $I_{4}$, with increasing values of $I_{4}$ for higher temperatures and the opposite tendency for $I_{3}$. The smaller free volumes, $R_{3}$, may correspond to holes in the crystalline regions connected with the aggregates of urethane urea groups, whereas the larger ones, $R_{4}$, might be ascribed to holes in amorphous regions. As compared to previous results in $[16,17], R_{3}$ has about the same value as the other polyurethane membranes for the same content of the soft segment. The decrease in $\tau_{4}$ with increasing PCL may be ascribed to an increase in cross-linking, resulting in a decrease in hole sizes. However, the rising tendency of $I_{4}$ with increasing PCL content means an increase in the hole density, so that an increase in porosity can be expected.

The temperature dependences of $R_{4}$ and $R_{3}$ are shown in Fig. 1b. The effect of temperature on $R_{4}$ is essentially the same for all PCL contents. For higher PCL content (15 to $25 \mathrm{wt} \%$ ) the $R_{4}$ radii are practically the same at each temperature, which might indicate a sort of saturation of the hole sizes due to a higher degree of cross-linking. As for $R_{3}$, it is apparent that the temperature dependence is attenuated for higher PCL concentrations, with stronger variations occurring for 5 and $10 \mathrm{wt} \%$ PCL contents only. For these lower concentrations, the smaller holes become even smaller with increasing temperature, suggesting that increasing temperature enhances the degree of cross-linking of the soft segments, a mechanism which seems to freeze for higher PCL content.

The $\mathrm{CO}_{2}$ permeability and the intensities $I_{3}$ and $I_{4}$ vs. PCL content at 298 $\mathrm{K}$ are shown in Fig. 1c. The permeability exhibits a tendency to increase with increasing PCL content. Both $I_{4}$ and $I_{3}$ increase with increasing PCL content, but in a different manner. For lower PCL concentration $I_{4}$ and the permeability go parallel, but at higher concentration $I_{3}$ follows the behaviour of the permeability. The FFV, combination of parameters $V_{\mathrm{f}}$ and $I$, was calculated for the smaller 


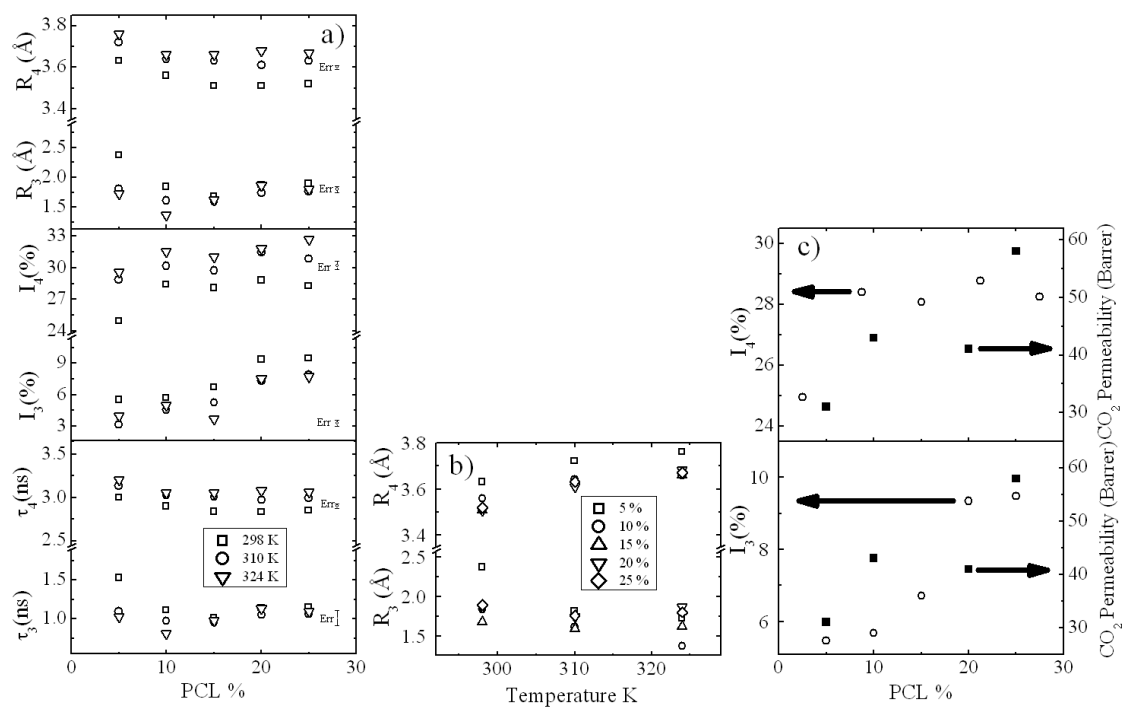

Fig. 1. (a) PALS parameters and the calculated radii of the Ps trapping sites as a function of PCL content for three different temperatures. (b) Temperature dependence of the free volume holes radii for different PCL content. (c) Relative intensities of the long lifetime components (o) and the $\mathrm{CO}_{2}$ permeability (-) observed at $298 \mathrm{~K}$ as a function of PCL content.

and the larger holes. A very weak dependence of the total free volume fraction, related to both kinds of holes, with $\mathrm{CO}_{2}$ permeability was observed, suggesting that, apart from the unoccupied space, other features are also important for the gas permeation (thus, involving actual transfer of matter), e.g., the connectivity of the voids. This observation is in agreement with results from amorphous polymers [13]. The permeability observed in these membranes is probably defined by an interplay of larger and smaller holes, the latter kind of "ingredients" probably increasing the interconnectivity. These membranes are phase separated with hard and soft segments. The expected changes of domain sizes and crystallinity may also contribute to the increase in permeability with PCL content. The observed enhancement of $o$-Ps intensity in the membranes with increasing PCL content is consistent with a reduction in the crystalline part of the membrane and a subsequent rise of the amorphous part. This effect may ease the gas diffusion path in the membranes, compensating even for an overall reduction in free-volume sizes, thus explaining the observed improvement in permeability with increasing PCL content.

For the same content of $\mathrm{PU}$, these membranes have lower $\mathrm{CO}_{2}$ permeability when compared to the membranes with soft segments PBDO [16] or PDMS [17]. The types of soft segments can also affect the permeation behaviour, because they could change the degree of microphase separation and the crystallinity of the 
membranes, questions which can still not be answered here but will be addressed later.

\section{Conclusions}

In bi-soft segment urethane/urea membranes studied by PALS, two types of free-volume holes of different sizes have been identified, corresponding probably to two separate domains of the soft and hard segments. In principle, the overall free volume in polymer membranes definitely influences the gas permeation properties. $\mathrm{CO}_{2}$ permeability was observed to be correlated to the density and size of both the larger and the smaller holes, the latter probably contributing to increased interconnectivity of the voids. The fractional free volume seen by the $o$-Ps may (and probably does) differ from the free volume involved in gas permeation. Positrons just experience an average static structural pattern, and do not feel forces due to concentration gradients that drive gas molecules across pathways through wobbled interconnections among the holes. The changes observed in the free-volume parameters obtained by PALS are smaller than those found in permeability. This is not fully surprising, as, e.g., the fractional free volume seen by o-Ps must exhibit a saturation limit when full trapping is reached, which is probably the case here. Studies on gas permeation [19] clearly indicate the influence of many factors, e. g. chain flexibility, chemistry at the free-volume walls, diffusion barriers, etc. The interconnectivity must play a crucial role in the gas permeation and definitely needs further systematic studies, by PALS and other techniques.

\section{Acknowledgments}

The authors gratefully acknowledge support received through the bilateral Hungarian-Portuguese Scientific exchange projects P-23/03 and P-23/05; M.F.F.M. thanks ISEC, too.

\section{References}

[1] A. Korematsu, Y. Takemoto, T. Nakaya, H. Inone, Biomaterials 23, 293 (2002).

[2] Principles and Application of Positron and Positronium Chemistry, Eds. Y.C. Jean, P.E. Mallon, D.M. Schrader, World Sci., Singapore 2003.

[3] Y.C. Jean, in: Positron Spectroscopy of Solids, Proc. Intern. School of Physics "Enrico Fermi", Course CXXV, 1993, Eds. A Dupasquier, A.P. Mills jr, IOS Press, Amsterdam 1995, p.563.

[4] G. Dlubek, H.M. Fretwell, M.A. Alam, Macromolecules 33, 187 (2000).

[5] G. Dlubek, J. Stejny, T.H. Luepke, D. Bamford, D. Petters, C.H. Huebner, M.A. Alam, M.J. Hill, J. Poly. Sci., Part B, Polym. Phys. 40, 65 (2002).

[6] D. Bamford, G. Dlubek, G. Dommet, S. Hoering, T. Luepke, D. Kilburn, M.A. Alam, Polymer 47, 3486 (2006).

[7] G. Dlubek, M.Q. Shaikh, R. Krause-Rehberg, M. Paluch, J. Chem. Phys. 126, 024906 (2007). 
[8] S.J. Tao, J. Chem. Phys. 56, 5499 (1972).

[9] M. Eldrup, D. Lightbody, J.N. Sherwood, Chem. Phys. 63, 51 (1981).

[10] D.W. Gidley, W.E. Frieze, A.F. Yee, E.T. Ryan, H.M. Ho, Phys. Rev. B 60, R5157 (1999).

[11] T. Goworek, K. Ciesielski, B. Jasińska, J. Wawryszczuk, Radiat. Phys. Chem. 58, 719 (2000).

[12] H. Nakanishi, S.J. Wang, Y.C. Jean, in: Positron Annihilation Studies of Fluids, Eds. S.C. Sharma, World Sci., Singapore 1988, p. 292.

[13] F.H.J. Maurer, M. Schmid. Radiat. Phys. Chem. 58, 509 (2000).

[14] M.F. Ferreira Marques, C. Lopes Gil, P.M. Gordo, A.P. de Lima, Zs. Kajcsos, D.P. Queiroz, M.N. de Pinho, Radiat. Phys. Chem. 68, 573 (2003).

[15] K. Kumar, Siddaramaiah, G.N. Kumaraswamy, H.B. Ravikumar, G. Ranganathaiah, Polym. Int. 54, 1401 (2005).

[16] M.F. Ferreira Marques, C. Lopes Gil, P.M. Gordo, A.P. de Lima, D. Placco Queiroz, M.N. de Pinho, Zs. Kajcsos, G. Duplâtre, Mater. Sci. Forum 445446, 289 (2004).

[17] M.F. Ferreira Marques, P.M. Gordo, Zs. Kajcsos, C. Lopes Gil, A.P. de Lima, D.P. Queiroz, M.N. de Pinho, Radiat. Phys. Chem. 76, 129 (2007).

[18] J. Kansy, Nucl. Instrum. Methods Phys. Res. A 374, 235 (1996).

[19] C. Nagel, K. Günther-Schade, D. Fritsch, T. Strunskus, F. Faupel, Macromolecules 35, 2071 (2002). 